\title{
Prospective, randomized, comparative study of delineation capability of radial scanning and curved linear array endoscopic ultrasound for the pancreaticobiliary region
}

Authors

Institutions

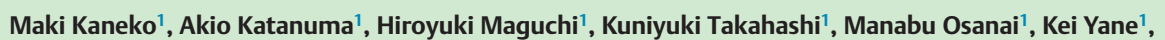
Syunpei Hashigo ${ }^{1}$, Ryo Harada ${ }^{1}$, Shin Kato ${ }^{1}$, Ryusuke Kato ${ }^{1}$, Masanori Nojima ${ }^{2,3}$

Institutions are listed at the end of article. submitted: 5. July 2013 accepted after revision: 26. May 2014

\section{Bibliography}

DOI http://dx.doi.org/

10.1055/s-0034-1377384

Published online: 10.7.2014

Endoscopy International Open 2014; 02: E160-E170

(c) Georg Thieme Verlag KG Stuttgart · New York

E-ISSN 2196-9736

\section{Corresponding author}

\section{Maki Kaneko, MD}

Center for Gastroenterology

Teine-Keijinkai Hospital

1-40-1-12 Maeda, Teine-ku

Sapporo

Hokkaido 006-8555

Japan

Fax: +81-11-6852967

maki.knk@gmail.com
Background and study aims: There are two types of endoscopic ultrasound (EUS) endoscope, the radial scanning (RS) and the curved linear array (CL). The type of EUS endoscope used at a first intent depends on local expertise, local habits and sometimes on how the examination is reimbursed. In Japan, RS is mainly used for observation, whereas CL is primarily used for histopathological diagnosis and treatment. We compared the imaging capabilities of RS and CL in evaluating the pancreaticobiliary region, a study which has not been performed previously.

Patients and methods: This prospective and randomized trial included 200 patients undergoing endoscopic ultrasonography of the pancreaticobiliary region by $R S(n=99)$ or $C L(n=101)$. The primary end point was the basal imaging capability of each technique. Eleven pancreaticobiliary areas were assessed and scored (range, $0-2$ ). Endoscopists evaluated each criterion, and a transcriber recorded the decisions in real time.

Results: The mean imaging scores in the RS and CL groups were 18.39 and 19.62 , respectively (significantly higher in CL, 95\%CI: $0.82-1.64$ ). Although no significant difference in imaging capability for

\section{Introduction}

Endoscopic ultrasonography (EUS) has become established for the identification of small lesions in the pancreaticobiliary region and for the detailed examination required for qualitative evaluation and differential diagnosis [1-7]. In the West, emphasis is placed on aspiration biopsy cytology (histology) [7 - 13], and curved linear array endoscopic ultrasonography (CL-EUS) has been widely adopted. Many centers only have one endoscope, and CL provides the possibility of performing diagnosis, fine-needle aspiration (FNA) and different kinds of therapy. In addition, shortening the duration of the examination and avoiding changing endoscopes are also important is- the pancreatic head, body, or tail was observed between $\mathrm{CL}$ and $\mathrm{RS}$, the imaging capability of $\mathrm{CL}$ for the pancreatic head-body transition region was superior to that of RS. Although no significant difference in imaging capability for the middle and inferior bile duct or the cystic duct was observed between CL and RS, the imaging capability of RS for the major duodenal papilla and gallbladder was superior to that of CL. For the area from the hepatic portal region to the superior bile duct, the imaging capability of CL was superior. In the delineation of the branch area of the celiac and superior mesenteric arteries, CL was also superior to RS.

Conclusions: The non-inferiority of the overall imaging capability of CL to that of RS was demonstrated. CL was superior in the delineation of the pancreatic head - body transition region, the area from the hepatic portal region to the superior bile duct, and the vascular bifurcation, whereas RS was superior in the delineation of the major duodenal papilla and gallbladder. Thus, for detailed evaluations of specific areas, the choice of scope should probably be considered.

sues in western countries. However, in Japan, because of concerns over tumor seeding [14] caused by tumor puncture and procedural accidents, endoscopic imaging diagnostic capabilities have been prioritized over histological analysis, and radial scanning endoscopic ultrasonography (RSEUS) is more widely used. Since the Japanese National Health Insurance program began reimbursing the technical fee for diagnostic EUS - FNA in Japan in April 2010, CL-EUS examinations and techniques have rapidly become more popular. Consequently, the number of cases in which an RS scope must be replaced by a CL scope for FNA has increased. If imaging capability does not differ between RS and CL, screening can be completed with one EUS scope. Although there are studies 


\begin{tabular}{|c|c|c|c|}
\hline $\begin{array}{l}\text { Score } \\
\text { Area }\end{array}$ & 2 & 1 & 0 \\
\hline $\mathrm{Ph}$ & $\begin{array}{l}\text { The region surrounded by SMA or SMV and the scope } \\
\text { is clearly visible }\end{array}$ & Partially visible & Not visible \\
\hline $\mathrm{Pb}$ & $\begin{array}{l}\text { The region between SPA or SPV and the gastric wall is } \\
\text { clearly visible }\end{array}$ & Partially visible & Not visible \\
\hline Phb & $\begin{array}{l}\text { The proximal parenchyma of the junction of SMV, } \\
\text { SPV, and PV is clearly visible }\end{array}$ & Partially visible & Not visible \\
\hline Pt & $\begin{array}{l}\text { The parenchyma adjacent to the splenic hilum and } \\
\text { left kidney is clearly visible }\end{array}$ & Partially visible & Not visible \\
\hline $\begin{array}{l}\text { Bile duct } \\
\text { (Bm and } \mathrm{Bi} \text { ) }\end{array}$ & The entire area is clearly visible & Partially visible & Not visible \\
\hline $\begin{array}{l}\text { Bile duct } \\
\text { (Bs and Bp) }\end{array}$ & The entire area is clearly visible & Partially visible & Not visible \\
\hline Cystic duct & $\begin{array}{l}\text { The spiral duct connected to the bile duct is clearly } \\
\text { visible }\end{array}$ & Partially visible & Not visible \\
\hline Gallbladder & $\begin{array}{l}\text { The gallbladder neck to the fundus is captured on a } \\
\text { single screen }\end{array}$ & Only the transverse image & Not visible \\
\hline Papilla & $\begin{array}{l}\text { Image of the pancreatic and bile ducts penetrating } \\
\text { the duodenal muscularis propria }\end{array}$ & $\begin{array}{l}\text { Image of only the pancreatic or bile duct penetrating } \\
\text { the duodenal muscularis propria or low-echoic } \\
\text { triangular lesion }\end{array}$ & Not visible \\
\hline CA & Clearly visible & - & Not visible \\
\hline SMA & Clearly visible & - & Not visible \\
\hline
\end{tabular}

Ph, pancreatic head; Pb, Pancreatic body; Phb, pancreatic neck; Pt, pancreatic tail; Bi, lower bile duct; Bm, middle bile duct; Bs, superior bile duct; Bp, hepatic portal region; CA, celiac artery; SMA, superior mesenteric artery; SMV, superior mesenteric vein; SPA, splenic artery; SPV, splenic vein.

on the basic imaging capability of RS [15], no study has compared the differences in imaging capabilities between RS and CL for lesions in the pancreaticobiliary region.

We conducted a prospective non-inferiority study comparing the imaging capabilities of CL and RS to investigate the suitability of $\mathrm{CL}$ as the standard for EUS examination in the pancreaticobiliary region.

\section{Patients and methods}

$\nabla$

\section{Patients}

This was a prospective, randomized, parallel-group study conducted at the Gastroenterology Center of Teine-Keijinkai Hospital. Consecutive cases requiring EUS examination, who had any biliopancreatic abnormality on ultrasound (US) and/or computed tomography (CT) between May and December 2011, were included. Exclusion criteria were (1) a history of surgical treatment for upper gastrointestinal or pancreaticobiliary disease; (2) suspected intestinal obstruction/stenosis; (3) lack of patient consent; (4) serious complications and unstable vital signs; and (5) judgment by attending endoscopists that examination would be unsafe. This study was approved by the ethics committee of Teine-Keijinkai Hospital, and informed, written consent was obtained from the patients. This trial was also registered with the University Hospital Medical Information Network (UMIN000007578, www.umin.ac. jp/ctr/index/htm).

\section{Instruments}

The EU-ME1 endoscopic ultrasound center (Olympus Medical Systems, Tokyo, Japan) was used along with the GF-UE260-AL5 RS scope (Olympus Medical Systems) and the GF-UCT240 or UCT260 CL scopes (Olympus Medical Systems). EUS images were in standard B-mode, and the frequency for both RS and CL was set at $6 \mathrm{MHz}$ which is the default setting for EUS and is generally used at our institution.

\section{Clinical protocol}

A third-party individual, who was different from the endoscopist performing the EUS, used biased-coin randomization to allocate cases to the groups. The patients were blinded about the scope used but the endoscopists were informed immediately before the examination. The patients were placed in the left lateral decubitus position, and the antispasmodic agent scopolamine butyl bromide $(20 \mathrm{mg})$ was administered intramuscularly. If scopolamine was contraindicated (patients aged $\geq 65$ years or those with heart disease, prostate disease, or glaucoma), 1 unit of glucagon was administered intramuscularly. Although, there is not enough evidence with regard to the use and administration methods of antispasmodic agents, we believe they are useful based on our observation of organs especially from the duodenum to the papilla. In addition, they are effective for a relatively long period of time, especially after intramuscular injection. For these reasons, they are administered routinely in our clinical practice. The sedative diazepam $(5 \mathrm{mg})$ and the analgesic pethidine hydrochloride $(35 \mathrm{mg})$ were administered intravenously, with additional doses of both being administered depending on patient consciousness levels. Forty minutes into the examination, if the images obtained using the chosen scope were considered insufficient, the scope was changed to the other type and the target area was reevaluated.

\section{Definitions and outcome measures}

The primary end point was the basic imaging capability to evaluate the pancreaticobiliary region. Eleven areas were assessed and each was assigned one of three scores: $0-2$ points. The summation of the 11 items was defined as the primary end point (imaging capability for observational index). $\odot$ Table 1 shows score definitions. "0" was assigned when imaging was impossible for 

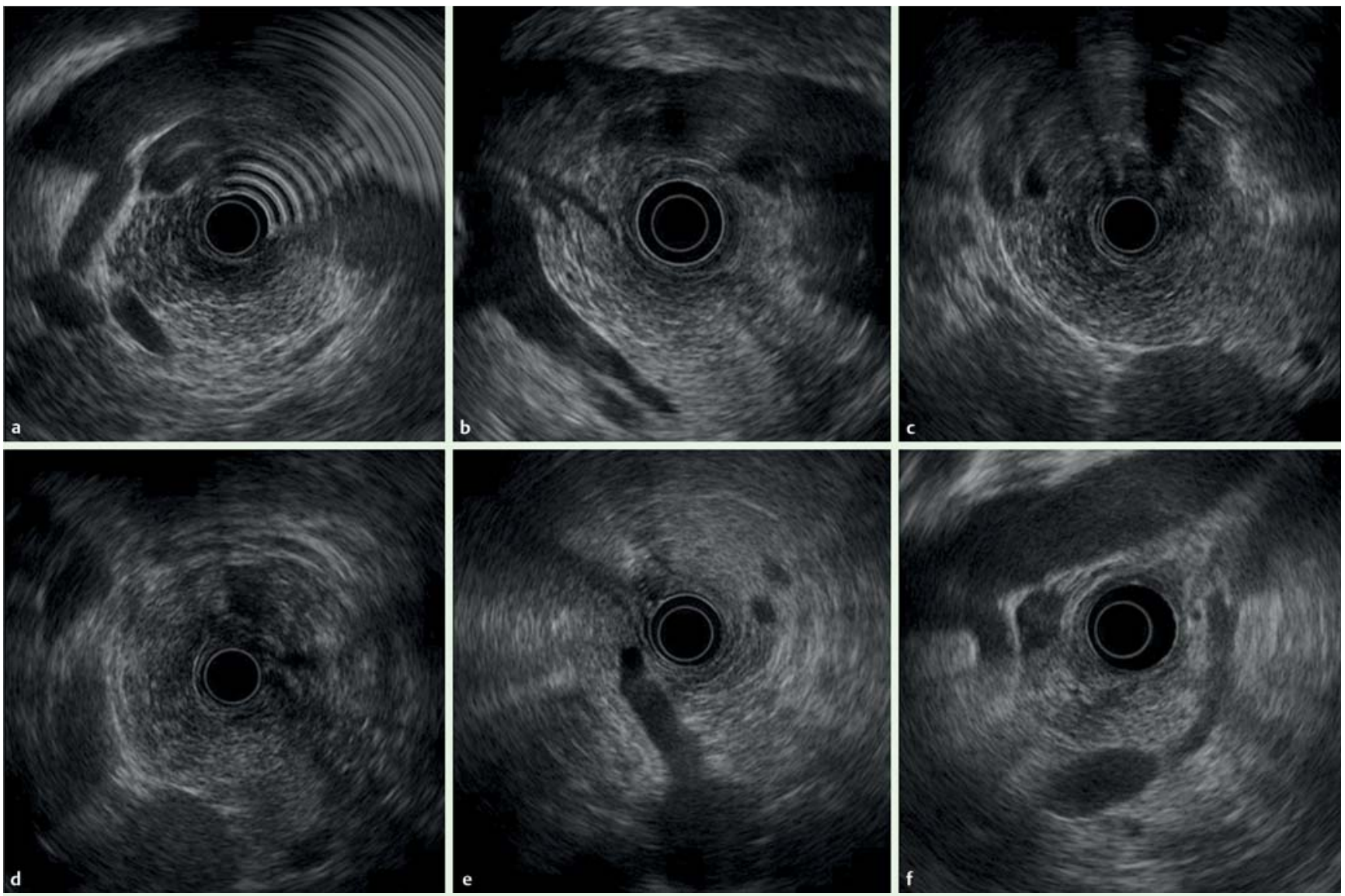

Fig. 1 Images with two-point score (pancreas, vasculature): RS group: A Pancreatic head B Pancreatic neck region C Pancreatic body D Pancreatic tail E Celiac artery branch $\mathrm{F}$ Superior mesenteric artery branch.

all organs. "1" or "2" were allocated according to standards determined for each imaged area. For the pancreas, "2" was assigned when the target organ was clearly visualized using imaging markers in each region and their positions relative to the pancreas were identified. The markers in each area and their relative positions were selected based on reports from the standard imaging techniques. The details are as follows $[16,17]$.

\section{Pancreas}

The pancreatic head $(\mathrm{Ph})$ was defined as the region between the superior mesenteric artery (SMA) or superior mesenteric vein (SMV) and the scope; the pancreatic body ( $\mathrm{Pb})$ as the region between the splenic artery (SPA) or splenic vein (SPV) and the gastric wall; the pancreatic neck (Phb) as the proximal parenchyma at the junction of SMV, SPV, and the portal vein (PV); and the pancreatic tail (Pt) as the parenchyma adjacent to the splenic hilum and left kidney, after confirming the splenic hilum by identifying SPV branches ( $\bullet$ Fig. 1,2). If the position of the pancreas relative to these markers or the pancreatic parenchyma was not visualized clearly for any portion, "1" was allocated.

\section{Bile duct}

Regions of the common bile duct were defined according to the classification by the Japanese Society of Biliary Surgery (JSBS), with the pancreatic portion of the common bile duct classified as the inferior bile duct (Bi) [18]. Although JSBS classifies the middle bile duct (Bm) and superior bile duct (Bs) as the upper and lower halves of the common bile duct segment from the convergence of the left and right bile ducts to the superior margin of the pancreas, we defined Bm as the common bile duct segment from the superior margin of the pancreas to the proximal right hepatic artery, and Bs as the common bile duct segment from the proximal right hepatic artery to the convergence of the left and right hepatic ducts. This is because EUS does not always clearly visualize the entire common bile duct. " 2 " was assigned when the segment between Bi and Bm was continuously and clearly visualized ( $\bullet$ Fig. 3,4 ), and " 1 " was assignment if it was only partially visualized (a score for "Bmi"). Moreover, to determine how well the long axis of the common bile duct was visualized on the same screen, the cases were categorized into three levels (two sectors or more, one sector only, or short axis only). "2" was assigned if the entire area from Bs to the hepatic portal region (Bp) was continuously visualized ( $\bullet$ Fig. 3,4 ), and "1" was assigned if it was only partially visualized (a score for "Bsp").

\section{Major duodenal papilla}

When evaluating the major duodenal papilla (the junction of the pancreatic and common bile ducts), the muscularis propria of the duodenum was identified. " 2 " was assigned if the penetration of the muscularis propria by both the common bile duct and pancreatic duct was visualized ( $\bullet$ Fig. 3,4), "1" was assigned if imaging showed penetration by only the pancreatic duct or only the common bile duct, or if only the hypoechoic proximal part of the papilla was visualized. 

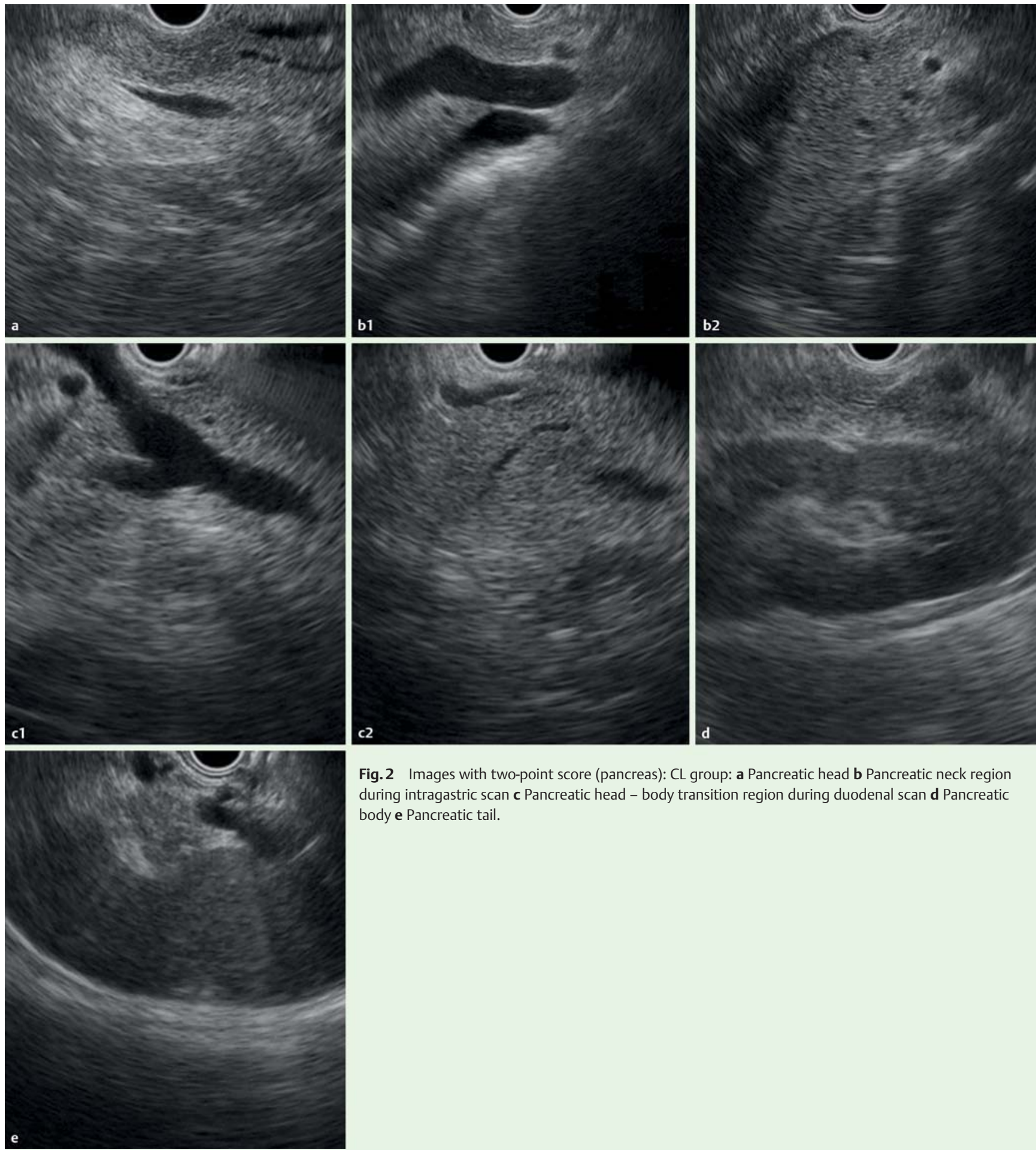

Fig. 2 Images with two-point score (pancreas): $\mathrm{CL}$ group: a Pancreatic head b Pancreatic neck region during intragastric scan c Pancreatic head - body transition region during duodenal scan $\mathbf{d}$ Pancreatic body e Pancreatic tail.

\section{Gallbladder}

For the gallbladder, "2" was assigned if the portion from the gallbladder neck to the fundus was captured on a single screen ( Fig. 3,5); "1" was assigned if only the transverse image was captured. In addition, "2" was assigned if the spiral valves (of Heister) were confirmed and if delineation was continuous to the gallbladder neck ( $\bullet$ Fig. 3,5), and "1" was assigned if delineation was only partial.
Arteries

When evaluating the celiac artery (CA) and SMA, "2" was assigned if the branching point of each artery from the aorta was clearly imaged ( $\bullet$ Fig. 1,5).

Secondary end points were determined by excluding the evaluations for CA and SMA, or CA, SMA, and Bsp from the primary end point score (nine items and eight items). Other secondary end points were scores for each evaluated area above, the imaging capability for lesions (lesions already identified by another imaging test) and identification of new lesions (lesions not identified by another test and visualized for the first time by EUS), examina- 

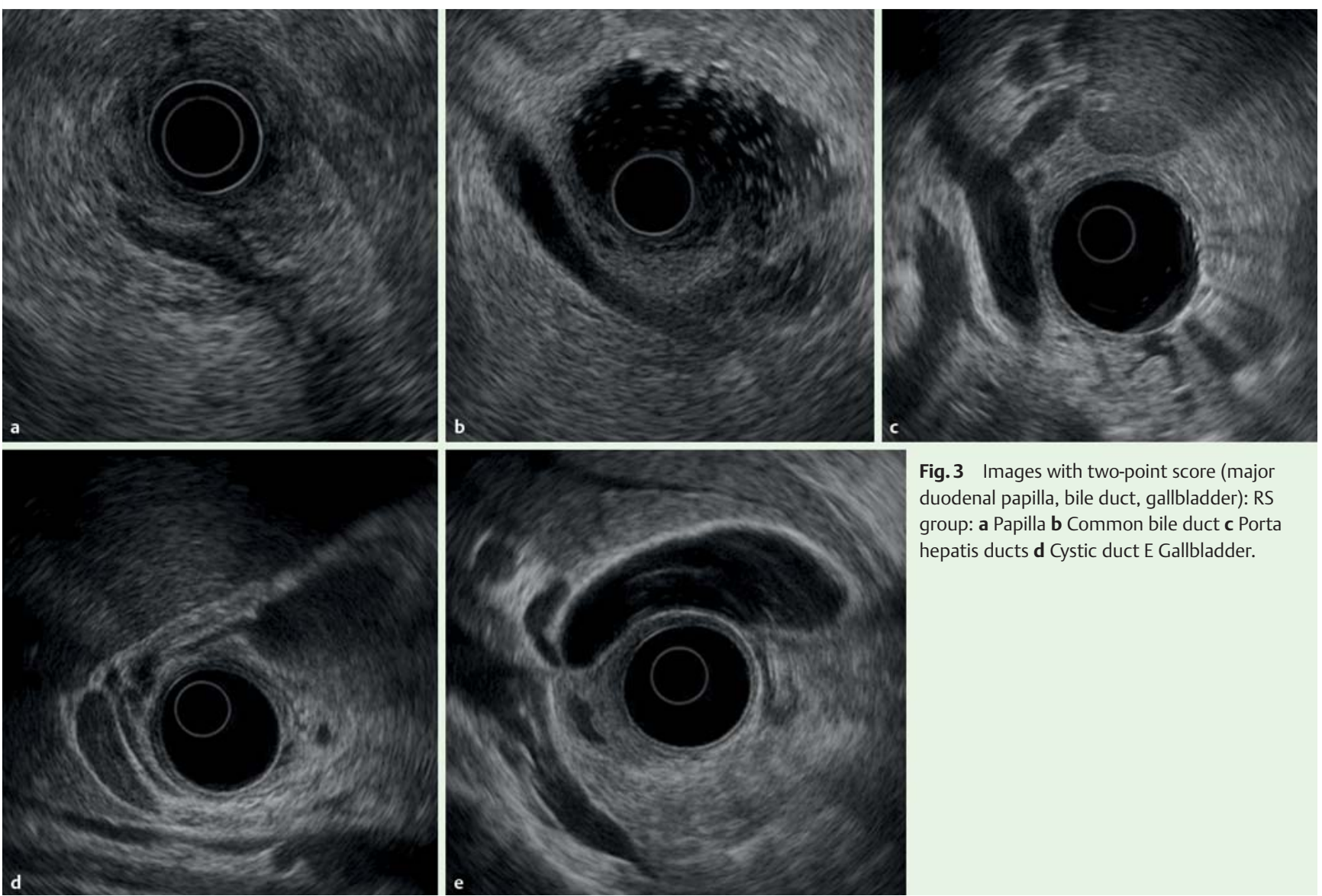

Fig. 3 Images with two-point score (major duodenal papilla, bile duct, gallbladder): RS group: a Papilla b Common bile duct c Porta hepatis ducts $\mathbf{d}$ Cystic duct $\mathbf{E}$ Gallbladder.

tion time, drug (sedative and analgesic) dose, and rate of scope change. These five items were compared between the groups.

\section{Operators and evaluators}

The endoscopists who performed EUS were 10 pancreaticobiliary endoscopy specialists and met all of the following essential criteria: $\geq 8$ years of endoscopy experience, $\geq 100$ EUS examinations/ year, an $\geq 200$ each of RS-EUS and CL-EUS examinations performed for more than 3 years. In addition, endoscopists who had performed $\geq 500$ RS-EUS examinations or $\geq 250$ CL-EUS examinations were classified as supervisors, and the other endoscopists were classified as experts. While there were four supervisors at our institution, examinations were always performed in the presence of at least one supervisor. On that basis, the endoscopists evaluated each criterion during the examinations, and a transcriber recorded their decisions in real time. Both the operators and supervisors evaluated all images. If they disagreed on the evaluation, the decisions made by the supervisors were given priority.

\section{Sample size calculation and statistical analysis}

A non-inferiority design was used in this study. The non-inferiority margin for the $\mathrm{CL}$ group in comparison with the RS group was $5 \%$ ( 1.1 points for 11 items) of the total points ( 22 points for 11 items). When calculating the difference in mean points between RS and CL groups, a lower limit of more than $-5 \%$ of the total points for the $95 \%$ confidence interval (CI) was considered to confirm the non-inferiority of the CL group. $P$ values for the difference in the mean points between RS and CL groups were calculated by $t$ test. We expected that the likelihood of each item being two points was 0.8 and that of each item being zero points was 0.2 for the sample size calculation. Its expectation and variance were estimated to be 1.6 and 0.64 , respectively. They were multiplied by the item total of 11 and the square root calculated, giving an estimated standard deviation of the total points of 2.65 for both groups. Similarly, the expectation of the total points was 17.6. Under these conditions, to maintain a one-sided type I error of $2.5 \%$ with a statistical power of $>80 \%$, a sample size of $>92$ cases per group is needed. Because we expected that a few cases would be excluded for some reason, 100 cases per group were enrolled. Despite the primary hypothesis of non-inferiority, the superiority of CL could be stated without any adjustment of the type I error, if a significant difference was observed.

For additional detailed analysis, we compared the proportions of achievement of two points in both groups for each of the 11 items using Fisher's exact test. If the proportions in the groups were $80 \%$ vs. $60 \%$, the statistical power was $84 \%$ for each analysis.

Usual inter- or intra-rater reliability of the scores used for the primary end point was difficult to perform because repetitive examinations for the same patients were not possible. Instead, we calculated coefficients of variation (CVs) for the scores stratified by experts and supervisors. The CV in this study can be interpreted as the consistency or stability of the observation among various patients. Since we basically investigated imaging capability in healthy regions, the scores should not be widely dispersed, apart from in patients with anatomical variations. In addition, we performed subgroup analysis according to the level of experience of the endosonographers as defined above. To control the potential for residual confounding of the primary end point evaluation, we performed multivariate analysis using general linear models ad- 


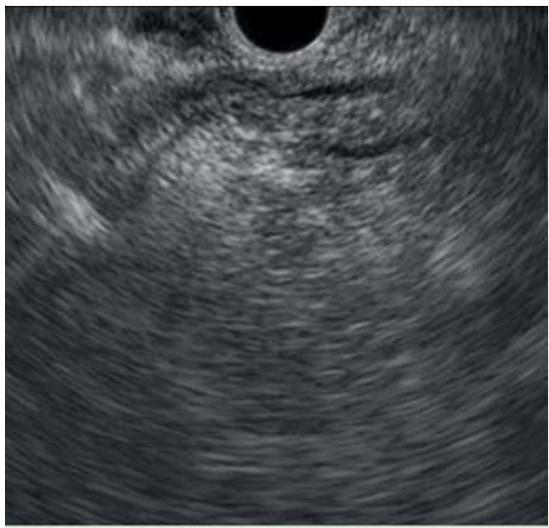

Fig.4 Images with twopoint score (major duodenal papilla, bile duct): $\mathrm{CL}$ group: a Major duodenal papilla b Common bile duct $\mathbf{c}$ The upper part of the common bile duct.
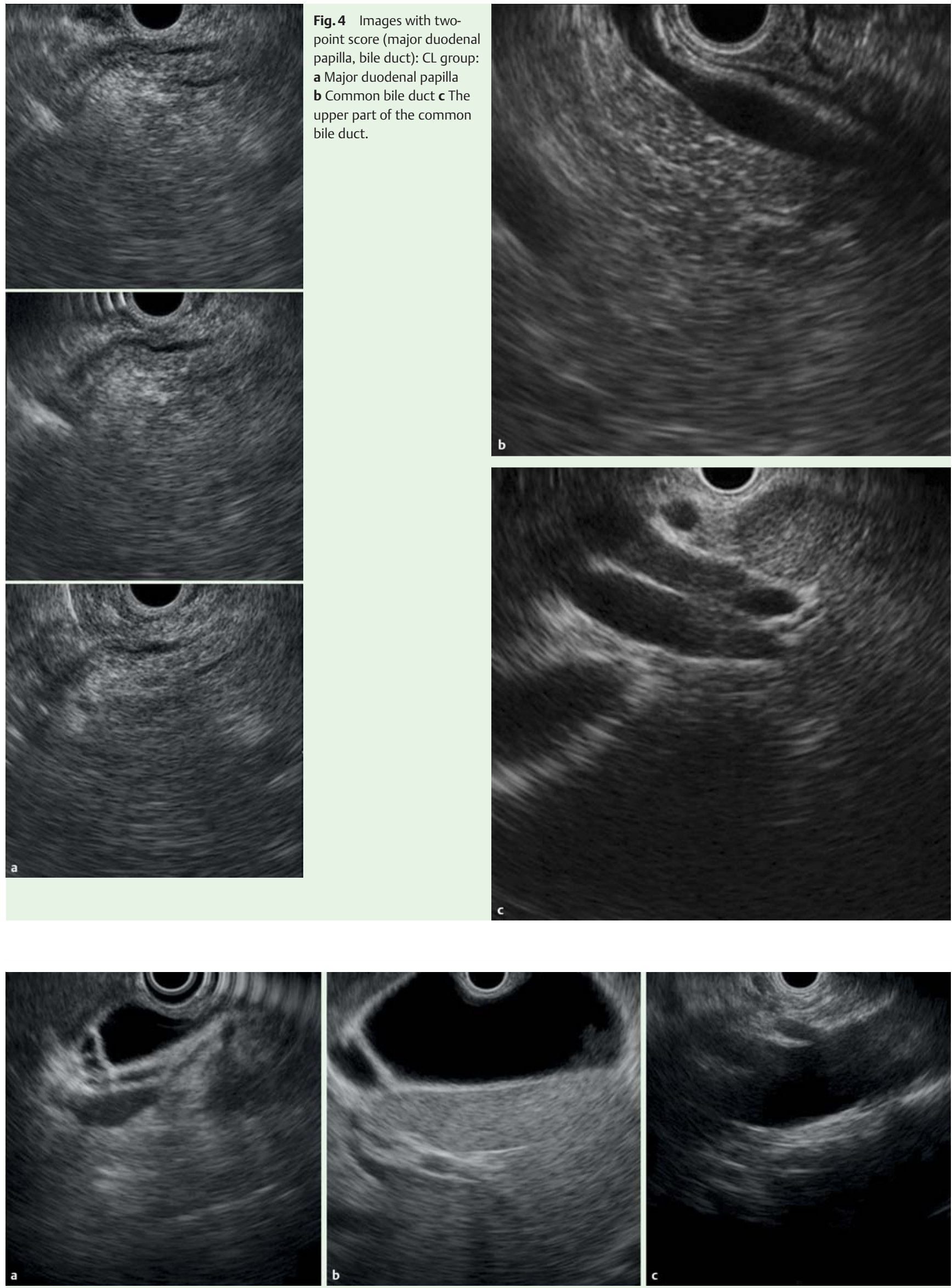

Fig.5 Images with two-point score (gallbladder, vasculature): CL group: A Cystic duct B Gallbladder C Celiac artery and superior mesenteric artery branches 


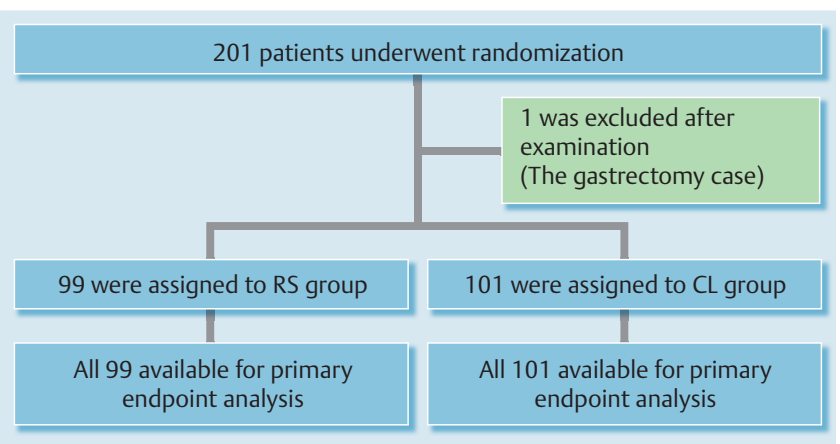

Fig. 6 Study enrollment and outcome.

justed by the level of experience and the chief purpose of the observation (background diseases).

For other analyses, $t$ tests were used for continuous values, whereas chi-square tests, Fisher's exact tests, or Mann - Whitney tests were used as required for discrete values. The sample size calculations were performed with the statistical tools provided by SWOG (http://www.swogstat.org/statoolsout.html) and all of the statistical tests were performed with SPSS 20 (IBM). $P$ values $<0.05$ were considered statistically significant.

\section{Results \\ $\nabla$}

\section{Patient characteristics}

A total of 201 consecutive cases were included. One was found to have undergone gastrectomy during examination and was thus excluded from the evaluation. The remaining 200 patients were divided randomly into two groups, with 99 in the RS group and 101 in the CL group ( $\bullet$ Fig. 6). There were no significant differences in the patient characteristics between both groups ( $\bullet$ Table $\mathbf{2}$ ).

\section{Primary end point}

Imaging capability for observational index

The results for the primary end point are shown in the upper row of $\bullet$ Table 3. When the overall scores for all 11 items were compared, the average score was found to be 18.39 for the RS group and 19.62 for the CL group (significantly higher in the CL group, $95 \% \mathrm{Cl}$ for the mean difference [MD] $0.82-1.64, P<0.001)$. These results confirmed the superiority of CL over RS.

\section{Secondary end points}

When items evaluating vasculature imaging at the branches of CA and SMA were excluded, the average scores for the remaining nine items for the RS and CL groups were 15.34 and 15.62 , respectively, with no significant difference ( $\bullet$ Table 3, 95\%CI for MD -0.06 to $0.62, P=0.103$ ); in addition, the CL group was found to be non-inferior to the RS group. However, when items evaluating Bsp were excluded, i.e., when only areas that could be previously observed by RS were analyzed, the average scores for the remaining eight items were 14.47 and 13.84 for the RS and CL groups, respectively, indicating the CL group to be inferior to the RS group ( $95 \% \mathrm{CI}$ for $\mathrm{MD}-0.92$ to $-0.35, P<0.001$ ).

\section{Capability of each area}

The ratio of two-point cases was used to compare the imaging capability for each area ( $\bullet$ Table 4 ). No significant difference in imaging capability for $\mathrm{Ph}, \mathrm{Pb}$, or $\mathrm{Pt}$ was observed between CL and RS. However, for delineation of the Phb, CL was superior to RS. In contrast, for the major duodenal papilla and gallbladder, CL was inferior to RS. In addition, no significant difference in imaging capability for the bile duct (Bmi) or cystic duct was observed between CL and RS. However, among the cases in which the bile duct was successfully visualized, the long axis imaging of $\geq 2$ bile duct sectors was possible in $87.9 \%$ of the RS group cases compared with $59.4 \%$ of the CL group cases, indicating that the CL was significantly $(P<0.001)$ inferior to RS ( $\bullet$ Table 5$)$. Furthermore, the RS group had a higher number of Bmi imaged, whereas the CL group had a larger number of Bsm imaged. For Bsp, CA, and SMA branch points, CL was superior.

\begin{tabular}{|llll|}
\hline Characteristics & RS group (n=99) & CL group (n=101) & P value \\
\hline Sex, male, no. (\%) & $65(65.6)$ & $58(57.4)$ & 0.248 \\
\hline Age, mean (SD), years & $61.9(11.8)$ & $63.4(14.0)$ & 0.398 \\
\hline Chief purpose of observation, no. & & & \\
\hline Pancreas & 7 & 12 & 0.246 \\
\hline Pancreas tumor & 15 & 12 & 0.499 \\
\hline Pancreas cyst including IPMN & 2 & 7 & 0.094 \\
\hline Main and/or branching pancreatic duct dilatation & 8 & 7 & 0.758 \\
\hline Pancreatitis & 4 & 2 & 0.393 \\
\hline Others & & & \\
\hline Biliary tract & 3 & 5 & 0.488 \\
\hline Bile duct stone & 1 & 1 & 0.989 \\
\hline Bile duct tumor & 36 & 41 & 0.539 \\
\hline Gall stone/acute cholecystitis & 0 & 2 & 0.159 \\
\hline GB tumor & 7 & 2 & 0.083 \\
\hline GB polyp & 7 & 5 & 0.528 \\
\hline Adenomyomatosis & 0 & 0 & $\mathrm{~N} / \mathrm{A}$ \\
\hline Ampullary tumor & 7 & 2 & 0.083 \\
\hline Abnormal levels of hepatobiliary enzymes & 2 & 3 & 0.667 \\
\hline Others & & & \\
\hline
\end{tabular}

Table 2 Baseline characteristics of patients.

IPMN, intraductal papillary mucinous neoplasm; SD, standard deviation; N/A, not applicable. 
Table 3 Capability for observation index: score.

\begin{tabular}{|c|c|c|c|c|c|c|c|c|c|c|}
\hline & \multirow[b]{2}{*}{ Group } & \multirow[b]{2}{*}{$\mathrm{n}$} & \multirow[b]{2}{*}{$\begin{array}{l}\text { Average } \\
\text { score }\end{array}$} & \multirow[b]{2}{*}{ SD } & \multirow[b]{2}{*}{ MD } & \multicolumn{2}{|l|}{$95 \% \mathrm{Cl}$} & \multirow{2}{*}{$\begin{array}{l}\text { Non- } \\
\text { inferiority } \\
\text { margin }\end{array}$} & \multirow[b]{2}{*}{$P$ value } & \multirow[b]{2}{*}{ Evaluation } \\
\hline & & & & & & $\begin{array}{l}\text { Lower } \\
\text { limit }\end{array}$ & $\begin{array}{l}\text { Upper } \\
\text { limit }\end{array}$ & & & \\
\hline \multirow[b]{2}{*}{ Overall } & RS & 99 & 18.39 & 1.74 & \multirow[b]{2}{*}{1.23} & \multirow[b]{2}{*}{0.82} & \multirow[b]{2}{*}{1.64} & \multirow[b]{2}{*}{-1.1} & \multirow[b]{2}{*}{$<0.001$} & \multirow{2}{*}{$\begin{array}{l}\text { Non-inferiority }{ }^{1} \\
\text { (suggesting } \\
\text { superiority }^{2} \text { ) }\end{array}$} \\
\hline & $\mathrm{CL}$ & 101 & 19.62 & 1.14 & & & & & & \\
\hline \multirow{2}{*}{ Excluding SMA and CA } & RS & 99 & 15.34 & 1.30 & \multirow{2}{*}{0.28} & \multirow{2}{*}{-0.06} & \multirow{2}{*}{0.62} & \multirow{2}{*}{-0.9} & \multirow{2}{*}{0.103} & \multirow{2}{*}{ Non-inferiority ${ }^{1}$} \\
\hline & $\mathrm{CL}$ & 101 & 15.62 & 1.14 & & & & & & \\
\hline \multirow{2}{*}{$\begin{array}{l}\text { Excluding } \\
\text { Bsp, SMA, and CA }\end{array}$} & RS & 99 & 14.47 & 1.05 & \multirow{2}{*}{-0.63} & \multirow{2}{*}{-0.92} & \multirow{2}{*}{-0.35} & \multirow{2}{*}{-0.8} & \multirow{2}{*}{$<0.001$} & \multirow{2}{*}{ Inferiority } \\
\hline & $\mathrm{CL}$ & 101 & 13.84 & 1.00 & & & & & & \\
\hline
\end{tabular}

SD, standard deviation; MD, mean difference; Cl, confidence interval; SMA, superior mesenteric artery; CA, celiac artery; Bsp, from the superior bile duct to the hepatic portal region.

${ }^{1}$ The lower limit of $95 \% \mathrm{Cl}$ was greater than the non-inferiority margin.

${ }^{2}$ The lower limit of $95 \% \mathrm{Cl}$ was greater than zero.

Table 4 Additional detailed analysis for comparison of the proportion achieving a two-point score in 11 items.

\begin{tabular}{|c|c|c|c|c|c|c|}
\hline & \multicolumn{2}{|l|}{$\begin{array}{l}\text { RS group } \\
(n=99)\end{array}$} & \multicolumn{4}{|l|}{$\begin{array}{l}\text { CL group } \\
(n=101)\end{array}$} \\
\hline & $\begin{array}{l}\text { Number achieving a } \\
\text { two-point score }\end{array}$ & $\%$ & $\begin{array}{l}\text { Number achieving } \\
\text { a two-point score }\end{array}$ & $\%$ & $\begin{array}{l}P \text { value (Fisher's } \\
\text { exact test) }\end{array}$ & Evaluation \\
\hline $\mathrm{Ph}$ & 99 & $100.0 \%$ & 97 & $96.0 \%$ & 0.121 & n.s. \\
\hline $\mathrm{Pb}$ & 99 & $100.0 \%$ & 101 & $100.0 \%$ & $\mathrm{~N} / \mathrm{A}$ & n.s. \\
\hline Phb & 88 & $88.9 \%$ & 99 & $98.0 \%$ & 0.010 & $S$ \\
\hline $\mathrm{Pt}$ & 99 & $100.0 \%$ & 100 & $99.0 \%$ & 1.000 & n.s. \\
\hline Papilla & 41 & $41.4 \%$ & 19 & $18.8 \%$ & 0.001 & 1 \\
\hline $\mathrm{BD}$ (Bmi) & 90 & $90.9 \%$ & 91 & $90.1 \%$ & 1.000 & n.s. \\
\hline $\mathrm{BD}(\mathrm{Bsp})$ & 7 & $7.1 \%$ & 79 & $78.2 \%$ & $<0.001$ & $S$ \\
\hline CD & 85 & $85.9 \%$ & 83 & $82.2 \%$ & 0.564 & n.s. \\
\hline GB & 47 & $47.5 \%$ & 13 & $12.9 \%$ & $<0.001$ & 1 \\
\hline CA & 89 & $89.9 \%$ & 101 & $100.0 \%$ & 0.001 & $S$ \\
\hline SMA & 62 & $62.6 \%$ & 101 & $100.0 \%$ & $<0.001$ & S \\
\hline
\end{tabular}

Ph, pancreatic head; Pb, pancreatic body; Phb, pancreatic neck; Pt, pancreatic tail; BD, bile duct; Bmi, from middle to lower bile duct; Bsp, from superior bile duct to the hepatic portal region; CD, cystic duct; GB, gallbladder; CA, celiac artery; SMA, superior mesenteric artery; N/A, not applicable; n.s., non-significant difference; I, inferiority; S, superiority.

Table 5 Comparative data: longitudinal view of the bile duct.

\begin{tabular}{|llll|}
\hline & Two or more regions & $\begin{array}{l}\text { One } \\
\text { region }\end{array}$ & $\begin{array}{l}\text { Short axis } \\
\text { only }\end{array}$ \\
\hline RS group, no. (\%) & $87(87.9)$ & $10(10.1)$ & $2(2.0)$ \\
\hline $\mathrm{CL}$ group, no. $(\%)$ & $60(59.4)$ & $27(26.7)$ & $14(13.9)$ \\
\hline
\end{tabular}

\section{Other secondary end points}

The results for secondary end points are shown in $\bullet$ Table6. RS visualized 168 of 170 lesions (98.8\%) and CL visualized 166 of $171(97.0 \%)$ clearly $(P=0.261)$. In addition, RS visualized 18 (18.2\%) new lesions, including seven cases of choledocholithiasis, one case of cholecystolithiasis, three gallbladder polyps, five cases of adenomyomatosis (ADM), one pancreatic tumor, and one pancreatic cyst. CL visualized 20 new lesions (19.8\%), including two cases of choledocholithiasis, four of cholecystolithiasis, four of gallbladder polyps (including one overlapping with cholecystolithiasis), seven cases of ADM, one pancreatic tumor, and three cases of pancreatic side-branch dilatation. No significant difference in the detection rate for new lesions was observed between the groups $(P=0.858)$.
Table6 Secondary end points.

\begin{tabular}{|llll|}
\hline & $\begin{array}{l}\text { RS group } \\
\text { (n=99) }\end{array}$ & $\begin{array}{l}\text { CL group } \\
\text { (n=101) }\end{array}$ & P value \\
\hline Lesion detectability, \% (no.) & $99.4(98)$ & $100(101)$ & 0.261 \\
\hline New lesion detectability, \% (no.) & $18.2(18)$ & $19.8(20)$ & 0.858 \\
\hline Examination time (min) & 28.5 & 31.8 & 0.005 \\
\hline Amount of sedative medicine (mg) & 12.7 & 14.3 & 0.028 \\
\hline $\begin{array}{l}\text { Amount of analgesic medicine } \\
\text { (mg) }\end{array}$ & 35.4 & 35 & 0.314 \\
\hline Scope change rate, \% (no.) & $0(0)$ & $7.9(8)$ & 0.007 \\
\hline
\end{tabular}

The average examination times were $28.5 \mathrm{~min}$ and $31.8 \mathrm{~min}$ for the RS and CL groups, respectively $(P=0.005)$. The sedative doses were $12.7 \mathrm{mg}$ and $14.3 \mathrm{mg}$ in the RS and CL groups, respectively $(P=0.028)$. There was no difference in analgesic dose.

The endoscopists elected to change the scope in eight cases, all of which involved changing CL to RS ( $\bullet$ Table 7). The main reasons for scope changes were as follows: uncertain positional relationship with the pancreatic lesion (three cases); difficulty in continuous visualization of the bile duct (major duodenal papilla to $\mathrm{Bi}$ ) (three cases) and cystic duct or gallbladder not detected (two 
Table 7 Cases with scope change.

\begin{tabular}{|llll|}
\hline No. & Lesion & Reason & Improved or no change \\
\hline 1 & Pancreatic tail pseudocyst & Uncertain cyst location & Improved \\
\hline 2 & Multiple IPMN & Widespread lesions; hence, uncertain position of each lesion & Improved \\
\hline 3 & AIP, BD stenosis & Incomplete continuous imaging of the bile duct & Improved \\
\hline 4 & Lower BD cancer & Incomplete continuous imaging of the bile duct & Improved \\
\hline 5 & GB swelling & Cystic duct not detected & Improved \\
\hline 6 & GB stones, ADM & Incomplete continuous longitudinal imaging of the gallbladder & Improved \\
\hline 7 & Pancreatic head cancer & Uncertain association between the tumor and main pancreatic duct & No change \\
\hline 8 & BD debris & Incomplete continuous imaging of the bile duct & No change \\
\hline
\end{tabular}

IPMN, intraductal papillary mucinous neoplasm; AIP, autoimmune pancreatitis; BD, bile duct; GB, gallbladder; ADM, adenomyomatosis.

cases). Visualization improved after a scope change in six cases (75\%).

There were no procedural adverse events in either group.

\section{Score reliability}

The results are shown in Supplementary Table S1. Using the RS scope, the experts performed 47 examinations, and the supervisors performed 52 examinations. Meanwhile, using the CL scope, the experts performed 41 examinations, and the supervisors performed 60 examinations. Briefly, the CVs for the overall observation scores were $8.0-10.5 \%$ in RS and $5.4-6.1 \%$ in CL. Since they were almost controlled within $10.0 \%$, they can be considered very stable.

\section{Supplementary stratified analysis and multivariate analysis}

As shown in Supplementary Table S2, the score defined as the primary end point was significantly higher for CL than for RS in each group ( Model 1). In addition, the difference in the scores between the CL and RS groups $(1.18, P<0.001)$ was consistent with the results in $\square$ Table3, even with the removal of the influence $(\checkmark$ Table S2, Model 2$)$.

\section{Discussion}

$\nabla$

EUS examination is used to identify small lesions and perform qualitative and differential diagnoses of lesions in the pancreaticobiliary region [1 1 7]. There are two types of scope, i.e. RS and CL. The advantages and disadvantages of each scope have previously been outlined on the basis of differences in the methods [15]. The wide $360^{\circ}$ scanning range of RS makes it easy to grasp relationships with surrounding organs and blood vessels, and it is comparatively easy to visualize imaging in alignment with the organ axis. However, its greatest disadvantage is that it cannot extract tissue samples. In contrast, although $\mathrm{CL}$ can collect tissue samples, its scanning range is narrow $\left(180^{\circ}\right)$, and it is difficult to align with the organ axis [5, 14]. EUS-FNA using a CL scope has been established as a useful examination technique not only in the West but also in Japan. However, only a few studies have compared the capacities of RS and CL to diagnose lesions in some specific organs such as the pancreas and esophagus [1921]. Moreover, no studies have compared the basic imaging capability of these methods in the pancreaticobiliary region. Therefore, the application of each scope for evaluating these lesions remains unclear. At our hospital, EUS has always played an important role in the pancreaticobiliary region [1], not only as a scanning technique for qualitative and differential diagnoses but also as an examination used when blood biochemical examinations find abnormal levels of pancreatic or hepatobiliary enzymes, and for identifying minute lesions when a small abnormality is found in the region by another imaging examination. In addition, sub-screening EUS examination (as preoperative examination for laparoscopic cholecystectomy) has been actively performed.

In this study, we evaluated imaging capability in the pancreaticobiliary region after selecting 11 evaluation items. The overall score for all 11 items was significantly higher in the CL group. However, when regions that are not primarily observed by RS, i. e. Bs-Bp, SMA, and CA, were excluded, the RS scores increased for the remaining eight items. In addition, when only vasculature evaluation is required after excluding SMA and CA, the remaining nine items exhibited nearly identical scores in both groups. The discrepancy between our non-inferiority hypothesis and the results could be explained by the marked superiority of CL for BsBp and SMA and the standard deviations being smaller than assumed. From these results, it can be concluded that, although imaging capability varied depending on the target area, there was no difference between groups for basic evaluation of the pancreaticobiliary region, excluding vasculature evaluation.

Assessment of imaging capability in each area revealed that $\mathrm{CL}$ was superior for Bs-Bp, Phb, and the branch areas of CA and SMA. Hara et al. reported the usefulness of CL for Bp imaging [22] and our study verified those findings. In contrast, for imaging of the major duodenal papilla and the gallbladder as well as

\begin{tabular}{|lllll|}
\hline & & Overall, \% & Excluding SMA, CA, \% & Excluding Bsp, SMA, CA, \% \\
\hline RS & Expert & 8.0 & 5.7 & 6.6 \\
\hline & Supervisor & 10.5 & 8.5 & 9.7 \\
\hline & Total & 9.4 & 7.3 & 8.3 \\
\hline CL & Expert & 5.4 & 6.5 & 6.7 \\
\hline & Supervisor & 6.1 & 7.6 & 7.7 \\
\hline
\end{tabular}

Supplementary Table S1 Coefficients of variation for the scores used for primary end point.

SMA, superior mesenteric artery; CA, celiac artery; Bsp, from the superior bile duct to the hepatic portal region; Expert: RS $<500$ and

$\mathrm{CL}<250$, Supervisor: $\mathrm{RS} \geq 500$ and $\mathrm{CL} \geq 250$. 


\begin{tabular}{|c|c|c|c|c|c|}
\hline \multirow[b]{2}{*}{ Strata } & \multirow[b]{2}{*}{ Comparison } & \multirow[b]{2}{*}{$\begin{array}{l}\text { Difference in the score } \\
\text { (11 items) }\end{array}$} & \multicolumn{2}{|l|}{$95 \% \mathrm{Cl}$} & \multirow[b]{2}{*}{$P$ value } \\
\hline & & & $\begin{array}{l}\text { Lower } \\
\text { limit }\end{array}$ & $\begin{array}{l}\text { Upper } \\
\text { limit }\end{array}$ & \\
\hline Expert & $C L-R S$ & 1.35 & 0.80 & 1.89 & $<0.001$ \\
\hline \multirow[t]{2}{*}{ Supervisor } & $\mathrm{CL}-\mathrm{RS}$ & 1.11 & 0.51 & 1.71 & 0.001 \\
\hline & & & & $P$ interaction value & 0.518 \\
\hline
\end{tabular}

$\mathrm{Cl}$, confidence interval; Expert: $\mathrm{RS}<500$ and $\mathrm{CL}<250$; Supervisor: $\mathrm{RS} \geq 500$ and $\mathrm{CL} \geq 250$.

\begin{tabular}{|c|c|c|c|c|c|}
\hline \multirow{3}{*}{ Comparison } & & \multirow{3}{*}{$\begin{array}{l}\text { Estimated difference in the score } \\
\text { (11 items) }\end{array}$} & \multicolumn{2}{|l|}{$95 \% \mathrm{Cl}$} & \multirow{3}{*}{$P$ value } \\
\hline & & & Lower & Upper & \\
\hline & & & limit & limit & \\
\hline \multirow{2}{*}{ Scope } & RS & vs. RS & Ref. & & \\
\hline & $\mathrm{CL}$ & 1.18 & 0.77 & 1.59 & $<0.001$ \\
\hline \multirow{2}{*}{ Experience } & Expert & vs. Expert & Ref. & & \\
\hline & Supervisor & 0.26 & -0.15 & 0.67 & 0.207 \\
\hline \multirow{10}{*}{$\begin{array}{l}\text { Chief purpose } \\
\text { of observation }\end{array}$} & GB stone/Acute cholecystitis & vs. GB stone & Ref. & & \\
\hline & Pancreas tumor & -0.30 & -1.03 & 0.43 & 0.413 \\
\hline & Pancreas cyst include IPMN & -1.09 & -1.72 & -0.45 & 0.001 \\
\hline & Pancreatitis & -0.31 & -1.11 & 0.49 & 0.444 \\
\hline & Pancreas others & -0.30 & -1.10 & 0.50 & 0.457 \\
\hline & Bile duct stone & -0.30 & -1.35 & 0.76 & 0.579 \\
\hline & Bile duct other & -0.77 & -1.89 & 0.35 & 0.177 \\
\hline & GB others & 0.51 & -0.41 & 1.43 & 0.273 \\
\hline & Adenomyomatosis & -0.14 & -1.02 & 0.74 & 0.759 \\
\hline & $\begin{array}{l}\text { Abnormal levels of hepatobiliary } \\
\text { enzymes }\end{array}$ & -0.91 & -1.92 & 0.10 & 0.077 \\
\hline
\end{tabular}

GB, gallbladder; IPMN, intraductal papillary mucinous neoplasm; Cl, confidence interval; Expert, $\mathrm{RS}<500$ and $\mathrm{CL}<250$; Supervisor, $\mathrm{RS} \geq 500$ and $\mathrm{CL} \geq 250$; Ref., reference.

long-axis visualization of the bile duct, CL was inferior. This may have been because of the differences in scope characteristics. For duodenal examination, because the tip of the scope is positioned in a relatively narrow space, the range of motion for the hard tip part is limited. To visualize the duodenal muscularis propria by $\mathrm{CL}$, the ultrasound scanning surface must be perfectly parallel to the muscularis propria, but in reality, this is difficult to achieve. Therefore, it was inferred that it would be easier to visualize the muscularis propria by RS. Although both scopes can visualize the proximal part of the papilla to some degree, CL was inferior for capturing images of the common bile and pancreatic ducts penetrating the duodenal muscularis propria. On the basis of the above results and because a detailed diagnosis of extent, including infiltration of the duodenal muscularis propria, is necessary, it appears that RS should be chosen over CL to perform an endoscopic papillectomy of papillary neoplasms.

Both scopes were able to visualize the middle and lower bile duct (Bmi) to the same degree. The benefit of the CL scope is its ability to visualize the upper bile duct (Bs), which is a characteristic feature of EUS. However, the RS scope is superior with regard to visualization of long-axis views of two or more segments (Bmi), and images can sometimes be easily recognized on the same screen. Lesion sites ranging from Bi to Bm may also be easily recognized.

With regard to gallbladder visualizing capability, CL was inferior. However, the detection rates were both low (RS, 47\%; CL, 13\%). This is because there was no landmark that could be used to visualize the gallbladder fundus, and two points based on the ob- tained long-axis views of the gallbladder were used to define the gallbladder fundus in this study. Further examination of zeropoint gallbladder images found that the detection rates for RS and CL were $0.0 \%$ and $0.9 \%$, with no significant difference between the groups. These results indicate that a series of shortaxis views was possible with both scopes. When observing the gallbladder by EUS, however, we should assess the gallbladder fundus carefully because there is no landmark to define this region, as described above.

There was no significant difference in the secondary end points of lesion imaging and new lesion imaging between the groups. However, examination time, overall sedative dose, and scope change rate tended to be greater for CL. One reason for this may be that when observing pancreatic head and bile duct lesions by $\mathrm{CL}$, the scope is often pushed into the duodenal bulb.

The limitations of this study include its single-center nature and the lack of assessment of the diagnostic capability. In the future, we aim to determine which scope would be more effective for detecting lesions, determining tumor extent, and making qualitative and differential diagnoses of tumors at each site.

This study has a large bias in some points, and this bias is not easily resolved. When this study was launched, we were concerned that the results might be biased by the differences in experience of each endoscopist operating the RS and CL scopes. Because RSEUS has been widely used in Japan, it is undeniable that the number of cases that endoscopists have experienced differs between RS and CL. However, the CL scope is also widely adopted in Japan, so endoscopists' skills operating the CL scope have improved. At 
our hospital, we have performed 700 - 800 RS-EUS examinations/ year since 1997 and $\geq 150-200$ CL-EUS examinations/year since 2006. Our endoscopists have acquired sufficient experience to perform the assessments of imaging capabilities in this study. Furthermore, examinations by both RS and CL were always performed in the presence of supervisors. As such, we believe that there was no difference in experience which would substantially affect the results of this study. In fact, the overall scores in the CL group exceeded those in the RS group, and the imaging capability scores for each area were comparable between groups.

In conclusion, our result demonstrated the superiority of the overall imaging capability of CL for the pancreaticobiliary region compared with RS. CL was superior in delineating the pancreatic head - body transition region, the area from the hepatic portal region to the superior bile duct, and the vascular bifurcation, whereas RS was superior in delineating the major duodenal papilla and the long axis of the bile duct/gallbladder. Thus, scope selection should probably consider the purpose of examination and the target area.

\section{Competing interests: None}

Institutions

${ }^{1}$ Center for Gastroenterology, Teine-Keijinkai Hospital, Sapporo, Hokkaido 006-8555, Japan

2 Department of Public Health, Sapporo Medical University School of Medicine, Sapporo, Hokkaido 006-8555, Japan

${ }^{3}$ Division of Advanced Medicine Promotion, The Advanced Clinical Research Center, The Institute of Medical Science, The University of Tokyo, Tokyo, Japan

\section{References}

1 Maguchi $H$. The roles of endoscopic ultrasonography in the diagnosis of pancreatic tumors. J Hepatobiliary Pancreat Surg 2004; 11: 1 - 3

2 Canto MI, Goggins M, Yeo CJ et al. Screening for pancreatic neoplasia in high-risk individuals: an EUS-based approach. Clin Gastroenterol Hepatol 2004; 2: 606-621

3 Liu CL, Lo CM, Chan JK et al. EUS for detection of occult cholelithiasis in patients with idiopathic pancreatitis. Gastrointest Endosc 2000; 51: $28-32$

4 Canto MI, Chak A, Stellato T et al. Endoscopic ultrasound versus cholangiography for the diagnosis of choledocholithiasis. Gastrointest Endosc 1998; 47: 439-448

5 Tierney WM, Adler DG, Chand B et al. Echoendoscopes. Gastrointest Endosc 2007; 66: $435-442$
6 Tierney WM, Kochman ML, Scheiman JM. Computed tomography versus endoscopic ultrasonography for staging of pancreatic cancer. Ann Intern Med 2005; 142: 590 - 591

7 Varadarajulu S, Wilcox CM, Eloubeidi MA. Impact of EUS in the evaluation of pancreaticobiliary disorders in children. Gastrointest Endosc 2005; 62: 239-244

8 Vilmann P, Jacobsen GK, Henriksen FW et al. Endoscopic ultrasonography with guided fine needle aspiration biopsy in pancreas disease. Gastrointest Endosc 1992; 38 : 172 - 173

9 Wiersema MJ, Kochman ML, Cramer HM et al. Endosonography-guided real-time fine-needle aspiration biopsy. Gastrointest Endosc 1994; 40: $700-707$

10 Chang KJ, Katz KD, Durbin TE et al. Endoscopic ultrasound-guided fineneedle aspiration. Gastrointest Endosc 1994; 40: 694-699

11 Chang KJ, Nguyen P, Erickson RA et al. The clinical utility of endoscopic ultrasound-guided fine-needle aspiration in the diagnosis and staging of pancreas carcinoma. Gastrointest Endosc 1997; 45: 387 - 393

12 Wiersema MJ, Vilmann P, Giovannini M et al. Endosonography-guided fine-needle aspiration biopsy: diagnostic accuracy and complication assessment. Gastroenterology 1997; 112: 1087-1095

13 Bhutani MS, Hawes RH, Baron PL et al. Endoscopic ultrasound guided fine needle aspiration of malignant pancreatic lesions. Endoscopy 1997; 29: $854-858$

14 Katanuma A, Maguchi H, Hashigo $S$ et al. Tumor seeding after endoscopic ultrasound-guided fine-needle aspiration of cancer in the body of the pancreas. Endoscopy 2012; 44: E160-E161

15 Katanuma A, Maguchi H, Osanai M et al. The difference in the capability of delineation between convex and radial arrayed echoendoscope for pancreas and biliary tract; Case reports from the standpoint of both convex and radial arrayed echoendoscope. Dig Endosc 2011; 23: 2-8

16 Fujita $N$, Inui $K$, Kida $M$ et al. Standard imaging techniques in the pancreatobiliary region using radial scanning endoscopic ultrasonography. Dig Endosc 2004; 16 : S118-S133

17 Yamao $K$, Irisawa $A$, Inoue $H$ et al. Standard imaging techniques of endoscopic ultrasound-guided fine-needle aspiration using a curved linear array echoendoscope. Dig Endosc 2007; 19: S180-S205

18 Japanese Society of Biliary Surgery. General rules for surgical and pathological studies on cancer of the biliary tract [Japanese]. 5th edn. Tokyo: Kanehara; 2003

19 Kochman ML, Elta GH, Bude $R$ et al. Utility of a linear array ultrasound endoscope in the evaluation of suspected pancreatic disease. J Gastrointest Surg 1998; 2: $217-222$

20 Gress $F$, Savides T, Cummings 0 et al. Radial scanning and linear array endosonography for staging pancreatic cancer: a prospective randomized comparison. Gastrointest Endosc 1997; 45: 138-142

21 Siemsen $M$, Svendsen $L B$, Knigge $U$ et al. A prospective randomized comparison of curved array and radial endoscopy in patients with esophageal cancer. Gastrointest Endosc 2003; 58: 671-676

22 Hara K, Bhatia $V$, Hijioka S et al. A convex EUS is useful to diagnose vascular invasion of cancer, especially hepatic hilar cancer. Dig Endosc 2012; $23: 26-28$ 